\section{Simultaneous tests and confidence intervals}

\section{KEVIN E. O'GRADY \\ University of Maryland, College Park, Maryland}

Following the rejection of an overall null hypothesis, it is often useful to locate those linear combination(s) of the parameters that have contributed to the rejection. Gabriel $(1968,1969)$ proposed a very general and widely applicable method, referred to as a Simultaneous Test Procedure (STP), that may be employed for such followup analyses in any of a variety of general linear model designs. Excellent discussions of various applications of this method can be found in Berger (1978), Harris (1985), Miller (1977), Morrison (1976), and Timm (1975). The purpose of the present program is to perform such STPs.

Estimation and Hypothesis Testing. Consider the following expression of the general linear models:

$$
\mathbf{Y}=\mathbf{X B}+\mathbf{E} \text {, }
$$

where $\mathbf{Y}$ is a matrix of $q$ random variables for $n$ observations (frequently referred to as "criterion variables," "dependent variables," etc.); $\mathbf{X}$ is a matrix of $p$ fixed and/or random variables for $n$ observations (often referred to as " "predictor variables," "independent variables," "grouping variables," "covariates," etc.); $\mathbf{B}$ is a matrix containing $p$ weights for each $\mathbf{Y}$ (variously referred to as "unstandardized partial regression coefficients," "estimates of effects," etc.); $\mathbf{E}$ is a matrix of $q$ random variables for $n$ observations, representing the errors in prediction.

Tests of hypotheses for this model can be determined from the hypotheses $(\mathbf{H})$ and error $(\mathbf{R})$ matrices:

$$
\left.\mathbf{H}=(\mathbf{A K B C})^{\prime}\left(\mathbf{A K}\left(\mathbf{X}^{\prime} \mathbf{X}\right)^{-1} \mathbf{K}^{\prime}\right) \mathbf{A}^{\prime}\right)^{-1}(\mathbf{A K B C}),
$$

where $\mathbf{A}$ is a matrix, each row of which contains contrasts on the estimates; $\mathbf{K}$ is an optional matrix, with rank less than or equal to $p$, which may be used to reparameterize the model; $\mathbf{C}$ is a matrix, each column of which contains weighting coefficients for the Ys; and the remaining matrices are defined as in Equation 1.

The definition of the error matrix depends, of course, on the assumption concerning the nature of the Xs. For a model in which all Xs are assumed fixed, $\mathbf{R}$ can be defined simply as:

$$
\mathbf{R}=\mathbf{E}^{\prime} \mathbf{E}
$$

Tests of significance can be determined from the roots of $\mathbf{R}^{-1} \mathbf{H}$. In the case of multiple Ys, Roy's greatest characteristic root test is performed. In the case of a single $\mathbf{Y}$, such a test can be reduced to the well-known $F$ test.

The support of the computer Science Center, University of Maryland is gratefully acknowledged. The author's mailing address is: Department of Psychology, University of Maryland, College Park, MD 20742.
For the case in which both $\mathbf{A}$ and $\mathbf{C}$ are vectors, it is also possible to determine simultaneous confidence intervals around a point estimate:

$$
\mathbf{a}^{\prime} \mathbf{K B c} \pm v \sqrt{\mathbf{c}^{\prime} \frac{\mathbf{R}}{d f_{e}} \mathbf{c}\left(\mathbf{a}^{\prime}\left(\mathbf{K}\left(\mathbf{X}^{\prime} \mathbf{X}\right)^{-1} \mathbf{K}^{\prime}\right) \mathbf{a}\right)},
$$

where $\mathrm{v}$ is a critical value (e.g., $t) ; \mathbf{X}^{\prime} \mathbf{X}$ is the uncorrected sums of squares and cross products of the $\mathbf{X}$ (its inverse is often referred to as the variance-covariance factors among the estimates); and the remaining matrices are defined as in Equation 1.

Input. Input to the program consists of: (1) a title card; (2) a parameter card containing information regarding the number of criteria, number of predictors, error term degrees of freedom, critical value for the construction of the confidence intervals (if such intervals are to be constructed), number of sets of contrast coefficient matrices to be read, number of sets of weighting coefficient matrices to be read, and the form of the various matrices input to the program; (3) either the error sums of squares and cross products matrix or the error variance-covariance matrix, in either lower triangular or full form; (4) either the predictor sums of squares and cross products matrix or the variance-covariance factors among the estimates matrix, in either lower triangular or full form; (5) a reparameterization matrix (optional); (6) the parameter estimates matrix; (7) a set of contrast coefficient matrices; (8) a set of weighting coefficient matrices (if omitted, all possible equally weighted linear combinations or compounds can be assessed).

Output. Summary output includes the job title, program options selected, error sums of squares and cross products matrix, error variance-covariance matrix, predictor sums of squares and cross products matrix (if input), variance-covariance factors among the estimates matrix, reparameterization matrix (if input), variance-covariance factors among the estimates determined from the reparameterization matrix (if a reparameterization matrix has been read), parameter esimates matrix, parameter estimates determined from the reparameterization matrix (if a reparameterization matrix has been read), contrast coefficients, and weighting coefficients. Printed output includes: for each multivariate hypothesis tested, the roots of $\mathbf{R}^{-1} \mathbf{H}$, canonical correlations, and Roy's greatest characteristic root test; for each univariate hypothesis tested, sums of squares and mean squares for hypotheses and error, $F$, and partial $R^{2}$; for each confidence interval drawn, the estimate associated with the confidence interval, its standard error, and its upper and lower bounds. A brief option is also available, which will eliminate all matrix output except the contrast and weighting matrices.

Technical Information. The program is written in 1977 ANSI standard FORTRAN IV and should be compatible with any modern FORTRAN compiler. All computations are performed in double-precision arithmetic, and core 
storage requirements on a UNIVAC $1100 / 92$ are approximately $53 \mathrm{~K}$ words. This includes $13 \mathrm{~K}$ words for program storage, and $40 \mathrm{~K}$ words for data storage.

Array storage consists entirely of a singly subscripted array stored in COMMON. This array is subdivided at execution time according to the problem size and the various program options chosen, through the use of integer pointers that allocate various portions of the array. Thus, the program is quite flexible in terms of the number of problems it can analyze. Because the various options affect storage allocation differently, both the type of problem analyzed and the options chosen determine the amount of core storage utilized. Moreover, the program reallocates memory at several stages during execution. Thus, it is extremely difficult to outline storage limitations. In any event, the size of all matrices involved in any given analysis may not exceed 20000 . This limitation can be easily altered by changing three statements in the MAIN routine.

In addition, two peripheral storage devices are used as binary scratch units. The unit numbers for these devices, as well as for the input unit, output unit, and up to three different alternate devices that might contain the error matrix, variance-covariance factors (or predictor sums of squares and cross products) matrix, and the parameter es- timates matrix, respectively, are assignable within the BLOCK DATA subprogram. Currently, these devices are assigned the numbers $2,3,5,6,0,0$, and 0 , respectively. The alternate input device(s) may be assigned unit numbers on the parameter card at execution time.

Availability. Reprints of this paper and an annotated source listing containing a user's manual can be obtained at no charge from the author.

\section{REFERENCES}

BERGER, M. P. F. (1978). A note on the use of simultaneous test procedures. Psychological Bulletin, 85, 895-897.

GABRIEL, K. R. (1968). Simultaneous test procedures in multivariate analysis of variance. Biometrika, 55, 489-504.

GABRIEL, K. R. (1969). Simultaneous test procedures-Some theory of multiple comparisons. Annals of Mathematical Statistics, 40, 224-250.

HARRIS, R. J. (1985). A primer of multivariate statistics (2nd ed.). New York: Academic Press.

MiLlER, R. G., JR. (1977). Simultaneous statistical inference (2nd ed.). New York: McGraw-Hill.

MoRrison, D. F. (1976). Multivariate statistical methods (2nd ed.). New York: McGraw-Hill.

TIMm, N. H. (1975). Multivariate analysis with applications in education and psychology. Monterey, CA: Brooks/Cole.

(Revision accepted for publication March 20, 1986.) 